\title{
A Practical Fuzzy Logic Controller for Sumo Robot Competition
}

\author{
Hamit Erdem \\ Baskent University, 06530 Ankara, Turkey \\ herdem@baskent.edu.tr \\ http://www. baskent.edu.tr/ herdem/
}

\begin{abstract}
This paper describes the design of fuzzy logic based sumo wrestling robot. The designed robot has a simple control algorithm and single fuzzy microcontroller is used in hardware implementation. The designed robot meets the specifications needed to compete in a sumo robot competition. The main difference of the designed system with earlier sumo robots is in control algorithms. A simple fuzzy logic controller is developed for detection and tracking of opponent in competition ring. Three infrared (IR) sharp sensors are used for target detection. Fuzzy microcontroller fuses the sensor data's and provides the necessary control signal to motors for heading robot toward the opponent. The fuzzy rules were optimized for the best results possible in software which are loaded in fuzzy controller. The implemented control algorithm shows better performance and executes the opponent detection algorithm in less time in comparison with conventional sumo robot algorithm. Design procedure and experimental results are presented to show the performance of the intelligent controller in designed system.
\end{abstract}

\section{Introduction}

Mobile robots provide an attractive platform for combining mechanical, electronic, computer, control and communication systems to create an integrated system for education and research. Mobile robots are mechanical devices capable of moving in an environment with a certain degree of autonomy. Autonomous navigation is associated with the availability of external sensors that capture information from the environment through different sensors. Motion autonomy in robotics may be defined as the ability for a robot to perform a given movement without any external intervention. Performing the task in best way is depended on performance of sensors and control algorithm. Fuzzy logic controller (FL) as nonlinear control method is used for sensor outputs reasoning and control of robot navigation in many works. FL is used to overcome the difficulties of modeling the unstructured, dynamically changing environment, which is difficult to express using mathematical equations [1/2/3. Fuzzy logic is simple sensor fusion method for combining sensor outputs. Sumo wrestling robot is one of important field to attract student attention for engineering science. The Sumo Robot competition has become more popular around the world 45. Similar to traditional sumo wrestling, the main objective is to force the competitor out of the

A. Ghosh, R.K. De, and S.K. Pal (Eds.): PReMI 2007, LNCS 4815, pp. $217225,2007$.

(C) Springer-Verlag Berlin Heidelberg 2007 
ring while staying in the ring. In sumo robot competition an autonomous mobile robot tries to find the opponent inside the wrestling board by using different sensors and force the opponent robot out of the ring. There are two main factors which effect wrestling result, faster opponent detection and applying more force to competitor. This work suggests FLC solution for first factor. This paper describes the design of a fuzzy logic controlled (FLC) sumo robot with considering the specifications needed to compete in a sumo robot competition. Fuzzy logic is used for opponent detection and tracking. This paper is organized as follows. Section II presents the Problem definition and discussion of sumo operation. Section III defines the developed control algorithm and FLC. Section IV describes the application of FLC to sumo robot and tracking of opponent problem. The performance of designed robot and applied control algorithm are discussed based on experimental results in last section.

\section{Problem Definition and Discussion of Sumo Operation}

Based on the specifications needed to compete in a sumo robot competition a simple mobile robot is designed. Generally there are four operation modes must be considered in design of sumo robot. These modes are;

Search or hunting mode: The robot moves around and its range sensors scans across the ring for sensing the opponent Opponent facing mode: The opponent has been sensed before and robot tries to face the opponent directly. Attack mode: Robot drive straight ahead at full power to push the opponent off the ring. Survive mode: The robot enters this state when it detects the ring border line. Its goal is to survive by not going off the competition ring.

In sumo robot competition, there are two main factor must be considered for wining. The first factor is the effective algorithm for facing and directing to target after sensing of opponent. The second factor is applying maximum power to motors during attack mode which dependent on motors power. This work suggests FLC solution for first factor. With consideration of above operation modes a sumo robot is designed. The main block diagram and structure of the robot are shown in Figure 1. Main parts of designed system are:

- Ring border line detection sensor (IR sensor)

- Opponent detection sensors (Sharp IR sensors: S1, S2, S3)

- Touch sensor (mechanical sensor)

- Right and left DC motors

- Main controller (ST52F513 fuzzy microcontroller)

- Motor driver

- Display (LCD)

Main controller receives inputs from touch, ring line border and target detection sensors and generate control signals for differential drive motion system. As shown in Figure 1, three Sharp GP2D12 infrared range sensors are used to sense the opponent. These sensors are mounted on the left, right and middle front of the robot. Target detection sensors generate an analog voltage. The voltage is 


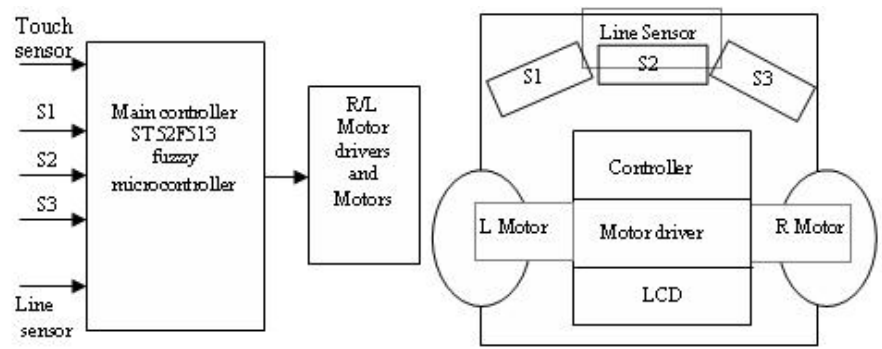

Fig. 1. Block diagram of designed robot and main structure of designed robot

higher the closer the sensor is to an object. Onboard ADC module of microcontroller is used for analog to digital conversion. Steering of robot is accomplished by rotating the motors at different angular velocities. When one motor rotates slower than the other, the robot will move in an arc curving to the side of the slower turning. By rotating the motors in the opposite direction of each other, the robot will rotate in place.

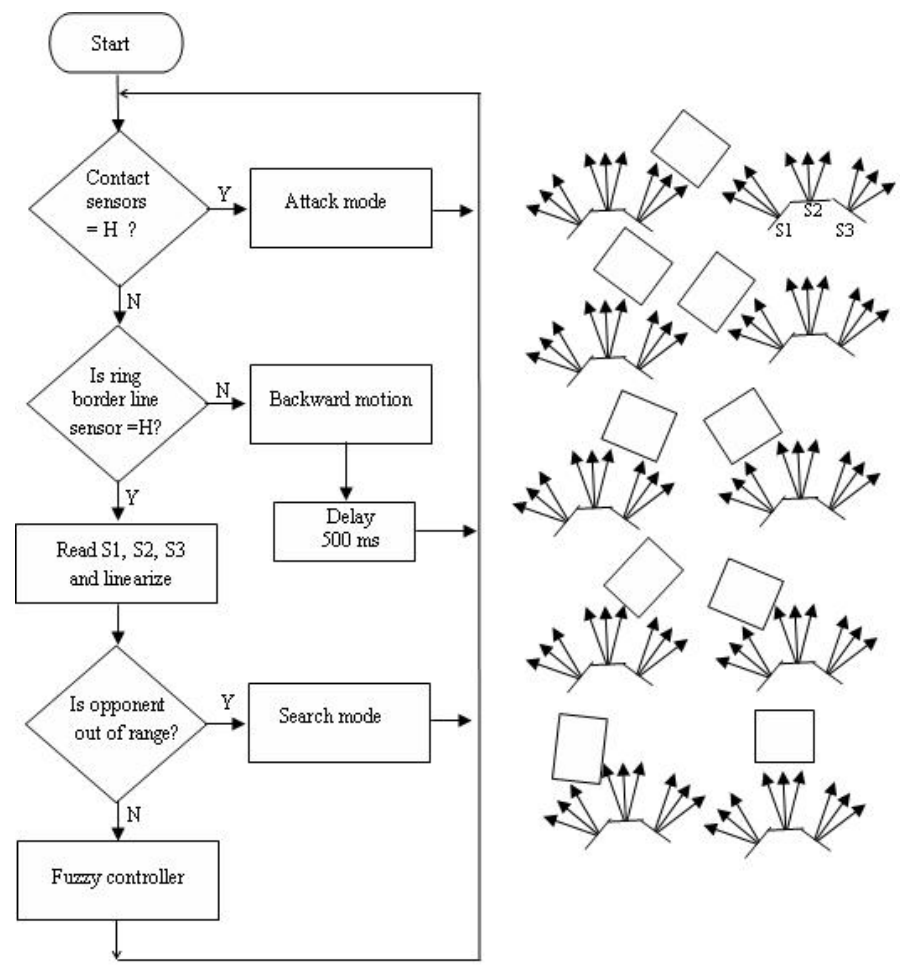

Fig. 2. Main control algorithm of sumo robot and some possible target positions 


\section{Main Control Algorithm}

Based on the operation modes of sumo robot and applying FLC for directing to target a control algorithm is developed. This algorithm is presented in Figure 2. As shown in algorithms of operation, in search mode, Robot rotates around itself and scans the area with IR sensors to find opponent. Right after sensing the target, robot tries to localize the opponent and turns toward it by changing angular speed of left and right motors. In attack mode robot applies maximum power to motors to attack opponent and pushes it to out of ring. Attack mode finishes with sensing of ring border line. In FLC section, there is a simple sensor fusion by fuzzy logic. Due to outputs of S1, S2, S3 IR sensors, robot tries to localize the opponent and attack to it.

\section{Opponent Detection Sensors}

For localization and detecting of opponent three GP2D12 IR Sharp range sensors are used. This sensor is a compact, self-contained IR ranging system incorporating an IR transmitter, receiver, optics, filter, detection, and amplification circuitry [6]. These sensors generate analog voltage for measured distance. The output voltage is 2.5 volt when an object is $10 \mathrm{~cm}$ away and 0.4 volts when an object is 80 centimeters away. The curve in Figure 3 shows relation of output voltage of sensor and measured distance.

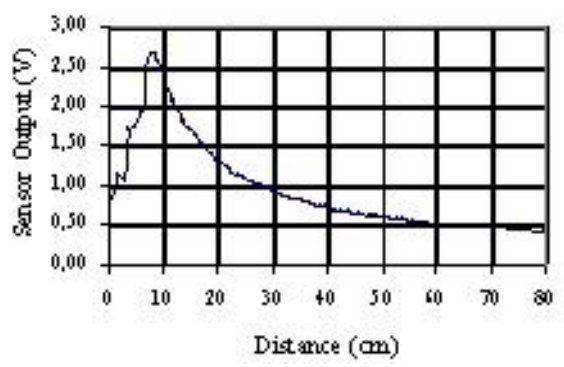

Fig. 3. Voltage versus range of the Sharp sensor

\section{Basic Concept of Fuzzy Control System}

A typical fuzzy control system consists of four components and the descriptions are stated as follows; 1) Fuzziffication: The fuzziffication interface performs a conversion from a crisp input value into a fuzzy set. In this application triangulation membership function (MF) is selected for input values fuzzification. 2) Knowledge Base: The knowledge base commonly consists of two sections: a database and a rule-base. The data base contains the MF of the fuzzy sets used in the fuzzy rules and the rule-base contains a number of fuzzy IF-THEN 
rules. 3) Inference Engine: The inference engine that performs the fuzzy reasoning upon the fuzzy control rules is the main component of the fuzzy controller. There are varieties of compositional methods in fuzzy inference, such as maxmin compositional operation and max-product compositional operation etc. 4) Defuzzification: The defuzzification converts the fuzzy output of the rule-base into a non-fuzzy value. The center of area (COA) is the often used method in defuzzification. In this application COA method is used for determination of real value for directing of motors. For onboard application, the selected controller supports directly the fuzzy inference method.

\subsection{Opponent Tracking with FLC}

Based on sumo navigation and competition rules an algorithms is developed. There is a complete algorithm in Figure 2 for robot navigation in sumo competition. The algorithm takes advantage of the essential characteristics of a differential drive to direct the wrestling robot. There are three IR sensors for detection of opponent position. A simple MISO system is considered for opponent detection and tracking. As shown in main algorithm, the role of FLC begins when one of the sensors receives signals from opponent. A FLC with three input and single output is considered. The reason behind choosing three MF for each input sensor variable is to limit the number of fuzzy rules. This is one of the important considerations in designing a fuzzy logic controller. Fewer rules improve system response time especially in practical application.

\subsection{Development of Fuzzy Rules}

Fuzzy rules are intuitive rules that can be driven by considering all possible scenarios with input sensor values. For example, if S1 and S2 detects weak signal and S3 detects medium signal, the logical action of the robot is to take a small turn to right. Robot sensing area and possible aspects of the sumo robot when

Table 1. Fuzzy rules for sumo robot

\begin{tabular}{|c|c|c|c|c|c|c|c|c|c|}
\hline Rule & S1 & S2 & S3 & Motion & Rule & S1 & S2 & S3 & Motion \\
\hline Rule1 & Low & Low & Medium & Small R. & Rule12 & Medium & Medium & High & Center \\
\hline Rule2 & Low & Low & High & Full R. & Rule13 & Medium & High & Low & Small L. \\
\hline Rule3 & Low & Medium & Low & Center & Rule14 & Medium & High & Medium & Center \\
\hline Rule4 & Low & Medium & Medium & Small R. & Rule15 & Medium & High & High & Center \\
\hline Rule5 & Low & Medium & High & Small R. & Rule16 & High & Low & Low & Full L. \\
\hline Rule6 & Low & High & Low & Center & Rule17 & High & Medium & Low & Small L. \\
\hline Rule7 & Low & High & Medium & Small R. & Rule18 & High & Medium & Medium & Center \\
\hline Rule8 & Low & High & High & Center & Rule19 & High & High & Low & Center \\
\hline Rule9 & Medium & Low & Low & Small L. & Rule20 & High & High & Medium & Center \\
\hline Rule10 & Medium & Medium & Low & Small L. & Rule21 & High & High & High & Center \\
\hline Rule11 & Medium & Medium & Medium & Center & & & & & \\
\hline
\end{tabular}



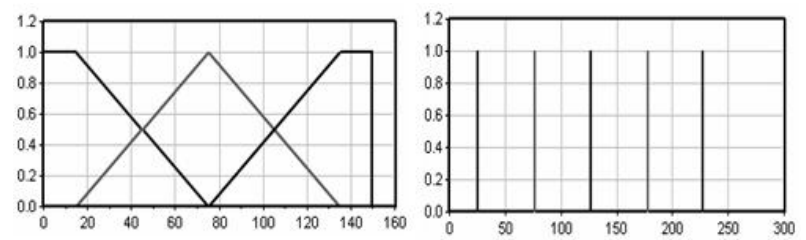

Fig. 4. Membership functions for input and output variables
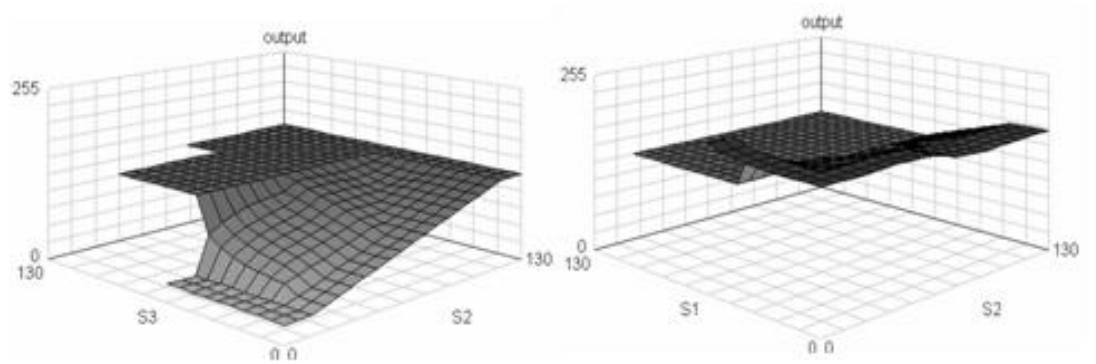

Fig. 5. Three dimensional plots for rule base (S2, S3 and output)(S1, S3 and output)

encountering an opponent are shown in Figure 2. Based on sumo behavior and possible aspects of the sumo robot when encountering an opponent, 21 rules are generated. The optimized rules for detection and tracking of target are shown in Table1.

For every input sensor three MF are selected, Low (L), Medium (M) and High (H) are fuzzy sets for input sensors. For output of fuzzy block singleton MF is selected. The selected membership functions for input and output variables are shown in Figure 4. IR sensors generate analog voltage approximately $0.42 \mathrm{~V}$ to $2.66 \mathrm{~V}$. This range is divided into three sets for every input sensor. For the output of FLC which controls the angular speed of motors five MF are considered. The five singleton membership functions are Full Left (FL), Small Left (SL), Center (C), Small Right (SL) and Full Right (FR). Based on output of fuzzy controller, two different PWM value are sent to motors. For example generation of Center $(\mathrm{C})$ value maintains the robot on a straight course while sending other values to controller will make robot turn to right or left.The result of fuzzy reasoning based on generated rules is evaluated and tested with using of Winfact 6 [7]. The Figure 5 shows three dimensional relations between sensors and fuzzy logic output.

\subsection{Main Controller}

In hardware application for onboard control, T52F513 fuzzy microcontroller is used [8]. T52F513 is a decision processor for the implementation of FL algorithms. This controller is a device of ST FIVE family of 8-bit Intelligent 
Controller Units (ICU), which can perform, Both Boolean and fuzzy algorithms in an efficient manner. Sensor outputs are sent to analog inputs of controller. Target detection sensors outputs are sent to fuzzy block after A/D conversion. The result of fuzzy reasoning is sent to PWM module for motor control. Based on fuzzy reasoning two different PWM value are send to motor drive circuit.

\section{Experimental Results}

The first application is obtaining sensors perception area. Three sensors are used for sensing target. It is considered that sumo robot is in motionless condition and a cubic object $(10 \times 10 \mathrm{~cm})$ is moving on horizontal direction from right to left with low speed. Due to the positions of Sharp sensors positions, firstly sensor S3 then sensor2 (S2) and finally sensor3 (S3) detects moving object nearly for 25 sampling time. The sampling time is 0.02s. Experiment condition and responses of sensors are shown in Figure 6. The sampling frequency of sensor values is $25 \mathrm{~Hz}$.

The second experiment is finding and pushing out the motionless object which is located at the back of robot. In start condition the head of the robot is against the object and sensors can not sense it. Robot starts in search mode and tries to find object. Firstly robot turns to left for a short time, the S1 detects the object. Based on control algorithm the rotating direction of motors changes until head of robot directed to the object, then it speeds up until hitting the object and pushes opponent out of the ring. For completely pushing out of ring, robot goes forward and backward due to sensor 2 response and hits the opponent several times. The steps of robot motion for detection of the opponent are shown in
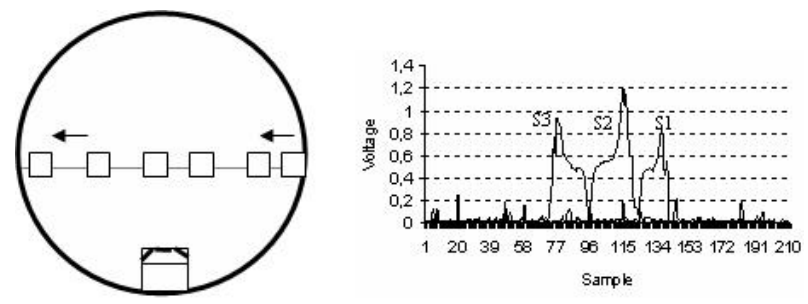

Fig. 6. Motionless robot and moving target from right to left
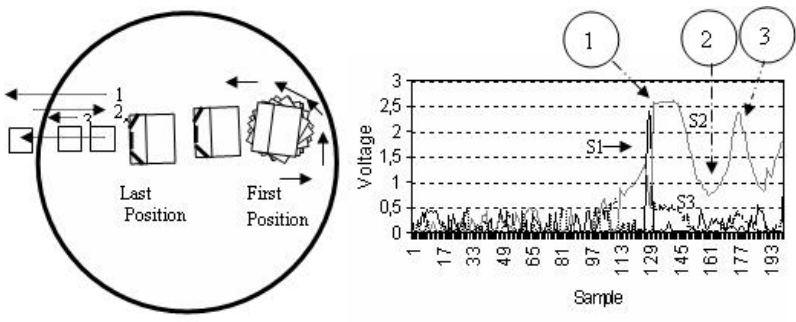

Fig. 7. Robot motions for attacking to target and sensors response 
Figure 7. The motion steps 1 to 3 in Figure shows the backward and forward motions of robot after it touches the opponen.

During rotational motion, opponent is detected by $\mathrm{S} 1$ for a short time, then robot rotate to left and tries to center to opponent. As shown in figur 8, S2 has the maximum detection time. Considering the sampling time which is $0.02 \mathrm{~s}$. $\mathrm{S} 2$ senses the opponent nearly for 10 sampling time (0.2s). Sensing of target by middle sensor (S2) is the biggest time for sensor in this experiment. This long time shows directing of robot towards the target. Changing of S2 output shows forward and backward motion of robot for pushing out the opponent. After sensing ring border line backward motion begins. The third experiment is detection of two different targets which are located in right and left of the robot. In starting motion robot rotates to left and senses the object one. With applying heading algorithm and attack mode pushes out object one and go to search mode and start left hand rotation. With sensing the next object applies the same algorithm and pushes out the second object. Figure 8 shows robot motions and Figure 9 shows the sensors outputs during the third experiment

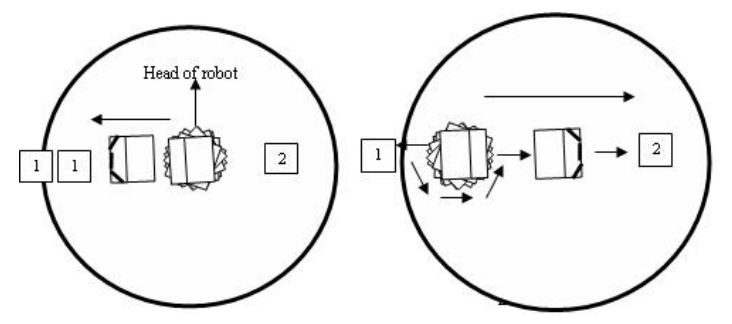

Fig. 8. Robot motions during attack to target 1 and 2

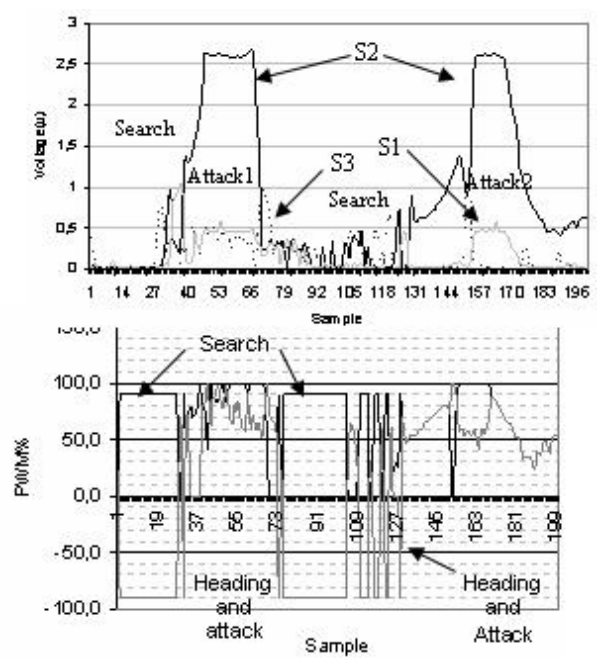

Fig. 9. Sensors responses in II experiment and PWM outputs for right and left motors 
and the applied PWM duty cycles to motor control circuit. The positive and negative values of PWM represent turning of robot in search mode.

\section{Conclusions and Results}

In this work, an intelligent sumo wrestling robot is designed. The designed robot has a very simple and minimal hardware configuration and meets the specifications needed to compete in a sumo robot competition. Fuzzy logic controller is used as sub controller for tracking of the opponent in a competition. In sumo competitions, fast sensing, localization and maneuvering in right time are important factors to win. In comparison with traditional sumo robot control algorithm, the designed system takes minimum time for execution of control algorithms in hardware application and can attack to opponent with a minimal maneuvers. The performance of applied control method can be improved with adding some extera sensors for sensing attack of the opponent during competion.

\section{References}

1. Driankov, D., Saffiotti, A.: Fuzzy Logic for Autonomous Vehicle Navigation. Springer, New York (2001)

2. Seraji, H., Howard, A.: Behavior-based robot navigation on challenging terrain: A fuzzy logic approach. IEEE Trans on Robot Automat 18(3), 308-321 (2002)

3. Vadakkepat, P., Miin, O., Peng, X., Lee, T.: Fuzzy behavior based control of mobile robots. IEEE Trans on Fuzzy Syst 12(4), 559-565 (2004)

4. Liu, J., Pok, C.K., Keung.: Learning: coordinated maneuvers in complex environments: a sumo experiment. In: CEC 99 (1999)

5. Liu, J.M., Zhang, S.W.: Multi-phase sumo maneuver learning. ROBOTICA 22(2), 61-75 (2004)

6. Sharp Corporation: Distance Measuring Sensor GP2D120. Spec. ED-99170 (99)

7. Winfact 6: Simulation program, http://www.kahlert.com

8. ST Microelectronics: ST52F513 sheet's. FUZZYSTUDIOTM5 User Manual ( 2001) 